\title{
Compact 13.5-nm free-electron laser for extreme ultraviolet lithography
}

\author{
Y. Socol, ${ }^{1, *}$ G. N. Kulipanov, ${ }^{2}$ A. N. Matveenko, ${ }^{3}$ O. A. Shevchenko, ${ }^{2}$ and N. A. Vinokurov ${ }^{2}$ \\ ${ }^{1}$ Falcon Analytics, P.O. Box 82, Netanya 42100, Israel \\ ${ }^{2}$ Budker Institute of Nuclear Physics, 630090, Novosibirsk, Russia \\ ${ }^{3}$ Helmholtz-Zentrum Berlin für Materialien und Energie GmbH, Berlin, Germany
}

(Received 28 October 2010; published 25 April 2011)

\begin{abstract}
Optical lithography has been actively used over the past decades to produce more and more dense integrated circuits. To keep with the pace of the miniaturization, light of shorter and shorter wavelength was used with time. The capabilities of the present 193-nm UV photolithography were expanded time after time, but it is now believed that further progress will require deployment of extreme ultraviolet (EUV) lithography based on the use of 13.5-nm radiation. However, presently no light source exists with sufficient average power to enable high-volume manufacturing. We report here the results of a study that shows the feasibility of a free-electron laser EUV source driven by a multiturn superconducting energyrecovery linac (ERL). The proposed $40 \times 20 \mathrm{~m}^{2}$ facility, using MW-scale consumption from the power grid, is estimated to provide about $5 \mathrm{~kW}$ of average EUV power. We elaborate the self-amplified spontaneous emission (SASE) option, which is presently technically feasible. A regenerative-amplifier option is also discussed. The proposed design is based on a short-period $(2-3 \mathrm{~cm})$ undulator. The corresponding electron beam energy is about $0.5-1.0 \mathrm{GeV}$. The proposed accelerator consists of a photoinjector, a booster, and a multiturn ERL.
\end{abstract}

DOI: 10.1103/PhysRevSTAB.14.040702

PACS numbers: 41.60.Cr, 85.40.Hp, 29.20.Ej

\section{INTRODUCTION}

Optical lithography has been used by the semiconductor industry over the past few decades to produce more and more fine features, and consequently more and more dense and powerful integrated circuits. On average, every two years the density of chip transistors doubled, as predicted by Moore [1] back in 1975 (Moore's law). To keep with the pace of the miniaturization, light of shorter and shorter wavelength was used with time. The capabilities of the present 193-nm UV photolithography (which is based on ArF excimer laser) were expanded time after time, but probably reached their physical limit. It is believed that further progress will require deployment of extreme ultraviolet lithography (EUVL) based on use of 13.5-nm radiation. Considerable effort was invested in the development of different aspects of the EUVL, including plasma-based sources of EUV radiation [2]. Particularly laser-produced plasma (LPP) sources for lithography are under rapid development and show essential progress. However, presently available LPP sources (from Cymer, Inc.) for lithography device (scanner) NXE:3100 from ASML Holding

\footnotetext{
*Corresponding author. socol@FalconAnalytics.com
}

Published by the American Physical Society under the terms of the Creative Commons Attribution 3.0 License. Further distribution of this work must maintain attribution to the author(s) and the published article's title, journal citation, and DOI.
$\mathrm{NV}$ operate at $11 \mathrm{~W}$ only-much below the industry demand of about 150-200 W EUV per scanner. The throughput of the NXE:3100 EUV scanner is therefore limited to 8-10 wafers per hour (WPH), while $100 \mathrm{WPH}$ is considered as a threshold for high-volume manufacturing. Therefore, a source with sufficient average power that enables EUVL high-volume manufacturing still has not been developed.

This study shows the feasibility of a free-electron laser (FEL) EUV source driven by a multiturn superconducting energy-recovery linac (ERL). While superconducting accelerator technology is complicated, it reached maturity with many machines functioning as user centers around the globe. The well-known examples include CEBAF, RHIC, and Cornell. The use of a high-gain FEL for the EUV lithography was proposed about a decade ago [3]. Here we tried to make some improvements of this approach. In particular, the use of a novel scheme of the multiturn ERL $[4,5]$ decreases the facility sizes and makes possible better control of beam dynamics and matching the ERL beam parameters with the FEL requirements (see, e.g., [6]). Optimizing e-beam parameters allows decreasing of the undulator length. Further size decrease and performance improvement may be achieved by the use of a regenerative amplifier FEL [7], which is also discussed below. It should be also mentioned that a recent Jefferson Lab proposal [8] for 10-100 eV photons FEL has for obvious reasons (100-eV photons correspond to $12.4-\mathrm{nm}$ wavelength) some similarity to the discussed design. 


\section{SASE FEL DESIGN CONSIDERATIONS}

\section{A. Electron beam energy and focusing}

To define the necessary electron energy $E=\gamma m c^{2}$, we use two equations. The first one is the undulator radiation wavelength,

$$
\lambda=\frac{d}{2 \gamma^{2}}\left(1+\frac{K^{2}}{2}\right)
$$

where $d$ is the undulator period, and

$$
K=e B_{0} d / 2 \pi m c^{2}
$$

(in the Gaussian unit system) is the undulator deflection parameter, proportional to the undulator field amplitude $B_{0}$. The second equation connects the undulator field amplitude $B_{0}$ with the magnet material coercivity $H_{c}$. For a planar hybrid undulator, $B_{0}$ may be estimated as

$$
B_{0} \approx H_{c} \frac{\sin (\pi \cdot t / d)}{\sinh (\pi \cdot g / d)},
$$

where $t$ is the thickness of the permanent magnet blocks (typically, $t \approx d / 3$, and we take this value below) and $g$ is the undulator gap. Some authors prefer to use the modified
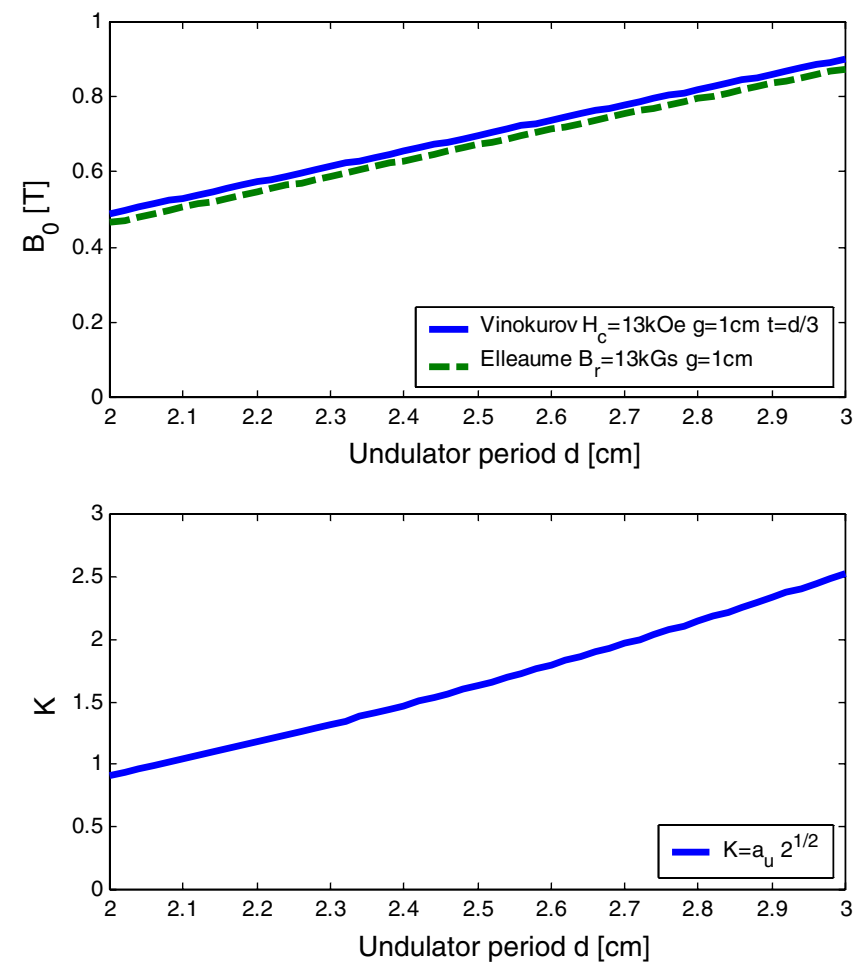

FIG. 1. Top: magnetic field amplitude $B_{0}$ for a planar hybrid undulator as a function of the undulator period $d$. $t$ is the thickness of the permanent magnet blocks $(t \approx d / 3$ is a typical value) and $g$ is the undulator gap. The results of the modified Halbach equation (Elleaume et al. [9]) are given for comparison. Bottom: the undulator parameter $K$.
Halbach equation (Elleaume et al. [9]) instead of Eq. (2), but it gives very similar results for short-period undulators $[10,11]$. The results are compared in Fig. 1 (we have scaled the original parameters to the coercivity $H_{c}=13 \mathrm{kOe}$ ).

For $K$ in the range 1.0-2.0 (higher $K$ values do not increase the FEL gain, as shown below-Figs. 4 and 5), the gap $g=1 \mathrm{~cm}$, and $H_{c}=13 \mathrm{kOe}$ (typical for $\mathrm{NdFeB}$ permanent magnets), Eq. (2) yields an undulator period $d$ in the range of about $2.0-3.0 \mathrm{~cm}$. Then Eq. (1) yields electron energies $E=500-1000 \mathrm{MeV}$. The resulting energy $E$ is given in Fig. 2 (top).

For such energies, to achieve lasing, one needs normalized rms emittance of below $\gamma \lambda / 4 \pi \sim 1.0-2.0 \mu \mathrm{m}(\gamma=$ $1000-2000, \lambda=13.5 \mathrm{~nm})$. Such values are achievable if bunch charge is less than $1 \mathrm{nC}$.

The natural vertical focusing of the undulator is described by the matched beta function $\beta_{u}=\sqrt{2} E /$ $e B_{0} \approx 5 \mathrm{~m}$. This value significantly exceeds the expected gain length of 1-2 m (see below, Figs. 4 and 5). Therefore, additional focusing would be beneficial. The focusing of the long undulator may be characterized by the average beta function $\bar{\beta}$. For the simplest case of the FODO lattice-equidistant sequence of focusing $(\mathrm{F})$ and defocusing (D) quadrupoles separated by nonfocusing (O) drift space- the minimum value of $\bar{\beta}$ is the double distance
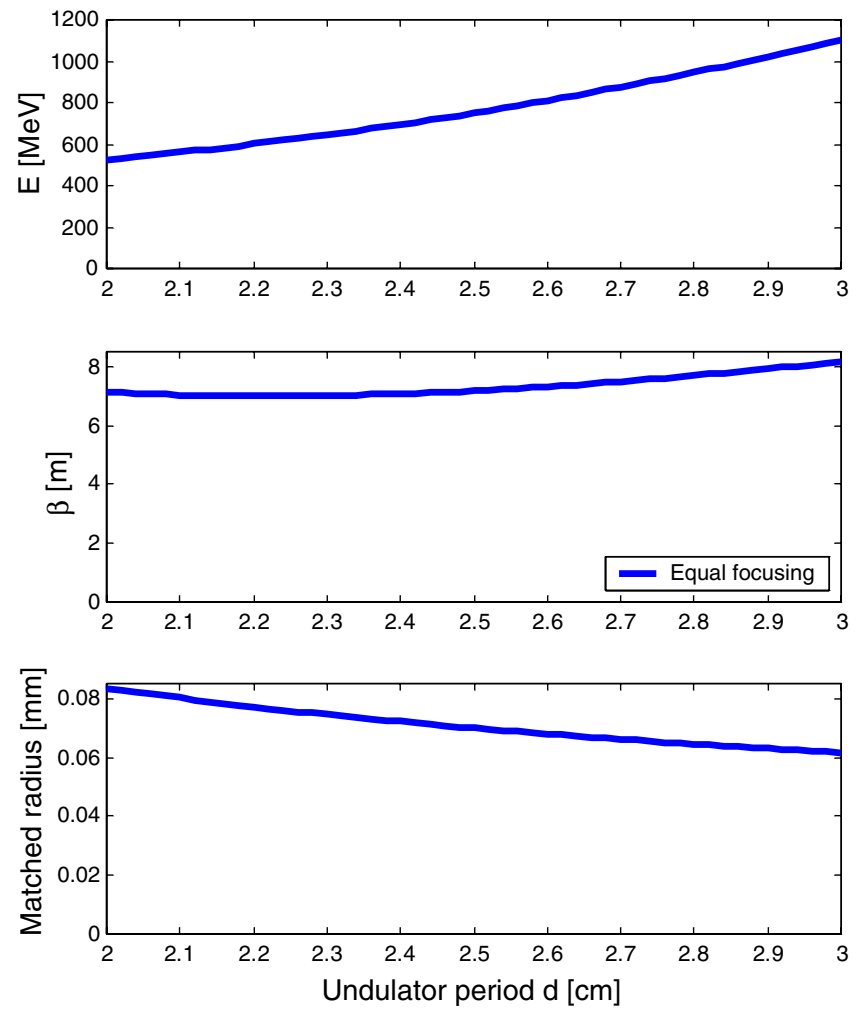

FIG. 2. Electron beam energy $E$, natural equal-focusing $\beta$ function, and matched beam radius $r_{0}$ as a function of the undulator period $d$. 
between the quadrupoles. Therefore, it is not easy to combine such strong focusing with the undulator field. For this reason one can try to use the natural undulator focusing, "redistributed" to the horizontal betatron oscillations by weak focusing quadrupoles. The natural equal-focusing $\beta$ function and matched electron beam radius $r_{0}$ are also presented in Fig. 2.

\section{B. Separate-tracks ERL}

During the commissioning of the first two-turn ERL [12] and the THz FEL driven by this ERL, several problems occurred. Many of them can be solved using the separatetracks ERL configuration [4,5]. The idea of this configuration was to separate the accelerated beam from the decelerated one. This could be achieved by using split accelerating structure, as it was done at CEBAF at Jefferson Lab.

A diagram for ERL with separated accelerated and decelerated beams is shown in Fig. 3. Electrons with injection energy $E_{0}$ passes through each rf accelerating section RF1 and RF2 twice, obtaining energy $E_{0}+4 \Delta E$. After that, the beam is used in the FEL undulator and enters the rf sections for deceleration. The last orbit length is chosen to tune the electrons' phase to the deceleration. Then after the first deceleration in RF2, electrons have energy $E_{0}+3 \Delta E$, which differ from energies $E_{0}+2 \Delta E$ and $E_{0}+4 \Delta E$ of the accelerated beams. Therefore, the separating magnet directs the decelerated beam to the appropriate arc. During further deceleration, the electron energy also differs from other beam energies by $\Delta E$. It is therefore possible to have only one beam at each arc, and one can adjust length, optics, and trajectory steering of each arc independently.

Splitting up the accelerating and decelerating beam lines has one major advantage: the optical requirements for accelerating and decelerating beam lines are very different. As the beam delivery system for FEL, the ERL must ensure emittance conservation and optimal bunching during acceleration. During deceleration, however, maximum energy acceptance with longitudinal "gymnastics" is required. Beam diagnostics is also simplified for separated

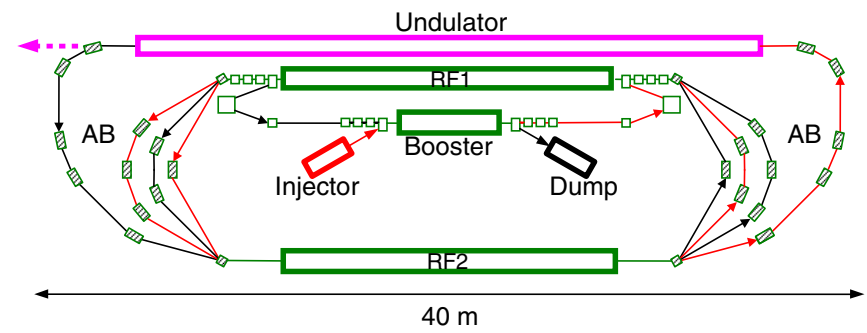

FIG. 3. The scheme of ERL with FEL. RF1 and RF2-rf accelerating/decelerating structures, $\mathrm{AB}$-achromatic bends. Red arrows-accelerating "fresh" beam, black arrowsdecelerating used beam. beams. Splitting of the rf system also decreases the length of sections with multiple beams, making the focusing issue less of a problem.

The above flexibility enables one to obtain femtosecond electron bunches in multiturn ERLs and use them to generate femtosecond $x$ rays and terahertz pulses.

The size of the ERL shown in Fig. 3 corresponds to the required electron energy of up to $1 \mathrm{GeV}$. The maximum energy gain $\Delta E$ per one linac is up to $250 \mathrm{MeV}$. The cascade injection with energies $40 \mathrm{MeV}$ (booster ERL) and $8 \mathrm{MeV}$ (injector) is proposed to be used for better adjustment of the electron beam parameters.

\section{FEL gain and power}

We considered that the full outer size of the installation will be $40 \mathrm{~m}$. Because of the space required by the beam bending, the undulator size has to be about $30 \mathrm{~m}$. For the SASE FEL it is generally assumed that the saturation is achieved after about 20 gain lengths [13]. Practically, this may be not enough to reach full saturation. For example, at the world-first x-ray FEL LCLS with $L_{g}=2.85 \mathrm{~m}$ the saturation (defined as beginning of the linear radiation power growth) was achieved at about $22 \times L_{g}$, while the saturated value of radiation power-at about $25 \times L_{g}$ [14]. Other experiments at LCLS with $L_{g}=3.3 \mathrm{~m}$ yielded saturation at about $20 \times L_{g}$ [15]. However, for our purpose of industrial ERL-based SASE FEL, achieving full saturation may be not optimal: at saturation, the electron beam energy spread considerably increases, which complicates the energy recovery. For an industrial machine, efficiency of energy recovery may well be more important than some fraction of the output power. In the following estimations we aim $L_{g} \leq 1.5 \mathrm{~m}$, keeping in mind options to slightly increase in the final design either the undulator length or the bunch current or both. The electron energy spread $\Delta \gamma$ must be less than $d / 4 \pi L_{g} \approx 1 \times 10^{-3}$ [16].

As mentioned above, the focusing of the long undulator may be characterized by the average beta function $\bar{\beta}$. Its optimal value is near the gain length. For the simplest case of the FODO lattice the minimum value of $\bar{\beta}$ is the double distance between the quadrupoles. Consequently, it is not easy to combine such strong focusing with the undulator field. One can therefore try to use the natural undulator focusing, redistributed to the horizontal betatron oscillations by weak focusing quadrupoles, as it was demonstrated at the first multisegment SASE FEL LEUTL [17]. In this case $\bar{\beta}=\sqrt{2} \beta_{u} \approx 7 \mathrm{~m}$.

As $\bar{\beta} \gg L_{g}$, for low enough energy spread and emittance one can use the simple expression for the gain length [18] to obtain the lower estimate of required peak current:

$$
L_{g}(1 \mathrm{D})=\frac{d}{4 \pi \sqrt{3} \rho}
$$

where the Pierce parameter $\rho$ 


$$
\rho=\frac{1}{\gamma}\left(\frac{I}{I_{A}} \frac{K^{2} d^{2} f^{2}}{64 \pi^{2} \varepsilon \bar{\beta}}\right)^{1 / 3},
$$

$I_{A}=m c^{3} / e \approx 17 \mathrm{kA}$ is the Alfvén current, $\varepsilon$ is the transverse rms emittance,

$$
f=\left[J_{0}\left(\frac{K^{2}}{4+2 K^{2}}\right)-J_{1}\left(\frac{K^{2}}{4+2 K^{2}}\right)\right]
$$

and $J$ are the Bessel functions.

For better accuracy we used Xie's parametric formula [18], which introduces 3D correction parameter $\Lambda$, while the actual gain length $L_{g}=L_{g}$ (3D) is connected with the 1D approximation $L_{g}(1 \mathrm{D})$ by

$$
L_{g}(3 \mathrm{D})=L_{g}(1 \mathrm{D}) \times(1+\Lambda) .
$$

The results are shown in Figs. 4 and 5. For the normalized emittance $\varepsilon \gamma=10^{-6} \mathrm{~m}$ and energy spread $\Delta \gamma=10^{-4}$, the peak current of $150 \mathrm{~A}$ is enough to achieve $L_{g} \leq 1.5 \mathrm{~m}$.

Estimating average power, we somewhat arbitrarily formulated the design goal as $5 \mathrm{~kW}$ of average EUV output. This estimation originated from the industry demand of about 200 W EUV per lithography device [2], multiplied
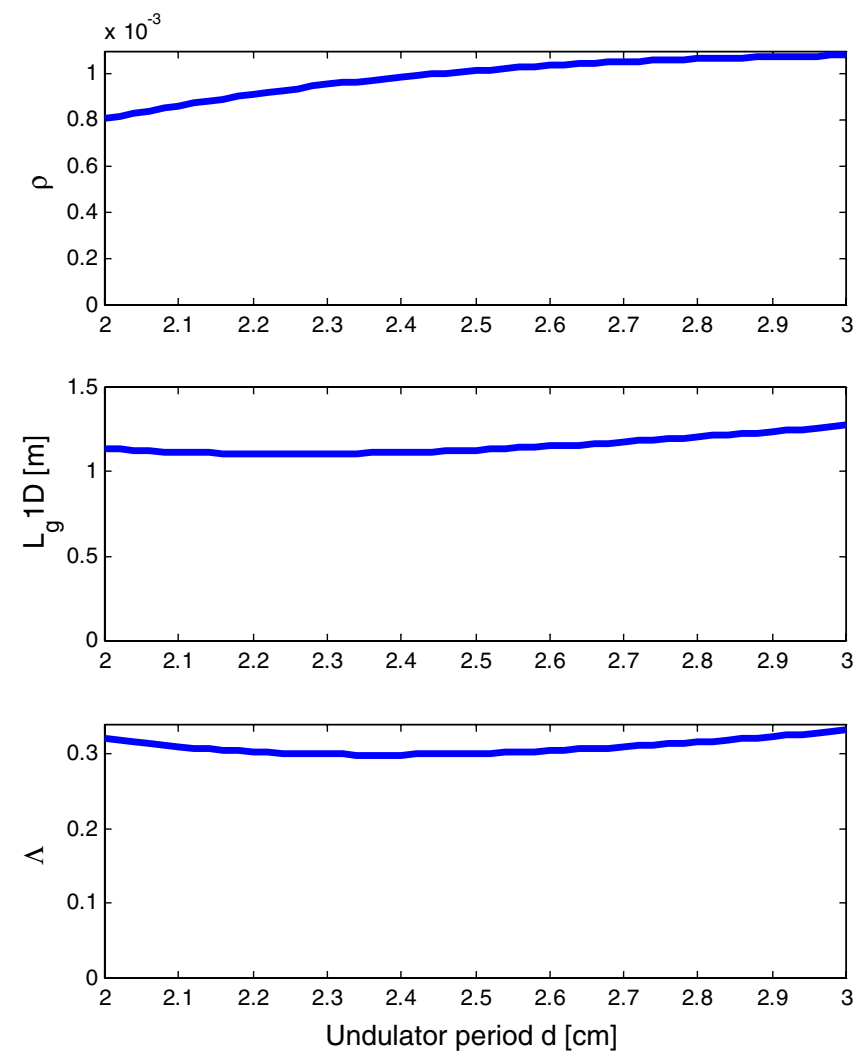

FIG. 4. The Pierce parameter $\rho$, $1 \mathrm{D}$ gain length $L_{g}$ (1D), and $3 \mathrm{D}$ correction parameter $\Lambda$ as functions of the undulator period $d$. Bunch current $I_{b}=150 \mathrm{~A}$, normalized emittance $\gamma \varepsilon_{\mathrm{rms}}=1 \mu \mathrm{m}$, energy spread $\Delta \gamma_{\mathrm{rms}}=0.02 \%$.
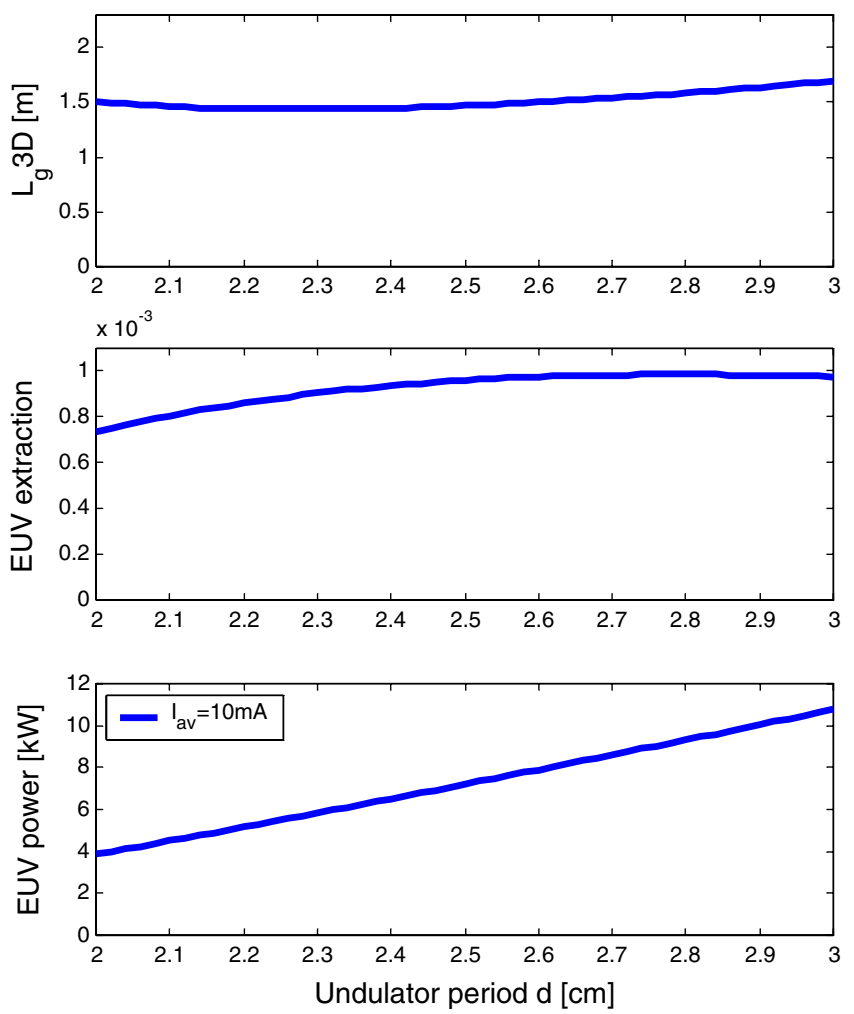

FIG. 5. Actual gain length $L_{g}$ (3D), EUV extraction efficiency, and extracted EUV power at $10 \mathrm{~mA}$ average current as functions of the undulator period $d$. Calculated according to parametric formula of Xie [18]. Bunch current $I_{b}=150 \mathrm{~A}$, normalized emittance $\gamma \varepsilon_{\mathrm{rms}}=1 \mu \mathrm{m}$, energy spread $\Delta \gamma_{\mathrm{rms}}=0.02 \%$.

by $10-12$ highest-resolution devices per semiconductor fabrication plant (fab), and by the additional factor of 2-3 to give considerable advantage to the FEL technology as opposed to the presently pursued plasma-based technology [2].

Since we imply energy recovery and low beam loss (of the order of $10^{-4}$ ), the $\mathrm{rf}$ power consumption will be determined to a large extent by the ratio of the energy of the dumped electron beam (not recovered) to the nominal energy. For $8 \mathrm{MeV}$ dump and $500 \mathrm{MeV}$ electron beam at FEL, the above ratio is $1.6 \%$ only. The FEL extraction efficiency is about [18] $d / 4 \pi L_{g} \approx 10^{-3}$, so we can expect the rf-to-EUV conversion to be $10^{-3} / 0.016 \sim 5 \%$. With the typical 50\% DC-to-rf efficiency, we still have $\sim 2.5 \%$ DC-to-EUV efficiency, leading to $200 \mathrm{~kW}$ estimation of input electric power. However, supporting systems demand additional power, and in our case these systems are expected to consume much more than the accelerating $\mathrm{rf}$ structure itself. Particularly, the $5000 \mathrm{~W}$ cryogenic plant uses on the order of $5 \mathrm{MW}$ of electricity continuously [19]. Therefore, cryogenic refrigeration is anticipated to be the main source of MW-scale power consumption of industrial EUV FEL.

For $5 \mathrm{~kW}$ average power, one needs beam average current of about $5-10 \mathrm{~mA}$. This average current was 
already reached at the Jefferson Lab ERL [8]. This indicates that proper electron optics is able to suppress electron beam instabilities at such currents. Choosing a modest value $0.1 \mathrm{nC}$ for the charge per bunch, one gets repetition frequency of $100 \mathrm{MHz}$, results reasonable for pulse lasers illuminating injecting photocathodes.

\section{OPTIONS: RAFEL AND STORAGE RING}

\section{A. Regenerative-amplifier FEL}

Further improvement of radiation parameters and decrease of the required peak current may be obtained using the regenerative-amplifier (RA) FEL [7]. The regenerative amplifier has a narrower and more stable radiation spectrum compared to the SASE case and allows for a shorter (7-10 $L_{g}$ ) undulator section.

Detail simulations of the RAFEL scheme are beyond the scope of this paper; however, simple estimations show that this scheme will reach saturation. For example, with the $9 \times L_{g}$ undulator we have a $10^{3}$ single-pass gain. If we outcouple, say, $5 \%$ of the output power into the feedback, we have still about $0.5 \%$ injection for the start of the next pass: the two EUV mirrors have reflectivity of $60 \%$ each [2], and we assumed additional reduction by a factor of 3 [7]. Therefore, the overall round-trip gain will be $10^{3} \times 0.005=5$ and we reach saturation in just a few round-trips.

The power density involved is rather high. Within the undulator the EUV beam is guided by and matched to the electron beam. Taking a typical value of electron beam rms radius $r_{0}=75 \mu \mathrm{m}$ (Fig. 2) and therefore the matched EUV beam waist $w_{0}=2 r_{0}=150 \mu \mathrm{m}$, we have the Rayleigh length $L_{R}\left(\pi w_{0}^{2}=\lambda \times L_{R}\right) L_{R} \approx 5.2 \mathrm{~m}$. At the distance of $1 \times L_{R}$ from the virtual waist, the EUV beam radius is $w=w_{0} \sqrt{2}$, and the effective area $\pi w^{2} / 2 \approx$ $7 \times 10^{-4} \mathrm{~cm}^{2}$. With $5 \mathrm{~kW} \mathrm{cw}$ EUV power, we have a power density of about $7 \mathrm{MW} / \mathrm{cm}^{2}$. While there are IR mirrors capable of bearing such loads [20], it is unpractical to assume that the same is achievable for EUV due to low mirror reflectivity (typically $60 \%$ or less).

Therefore there may be demand to use a ring resonator [21] as was done in the first RAFEL demonstration [7]. A mirror at grazing angle $\theta$ has the potential to withstand much higher EUV power, and not only due to the $\sin (\theta)$ factor of increased area of incidence. Really, according to Fresnel formulas (Ref. [22]) for refractive index $n=1+$ $1 \times 10^{-3}$ the normal-incidence reflectivity $[(n-1) /$ $(n+1)]^{2}$ is about $2.5 \times 10^{-7}$, but at grazing angle $\theta=$ $4^{\circ}$ the reflectivity grows to nearly $10^{-2}$. The corresponding amplitude reflectivity, the square root of the intensity reflectivity, will be therefore 0.1, comparable with normalincidence reflectivity in IR and visible [e.g., for $n=1.5$, normal-incidence amplitude reflectivity $(n-1) /(n+1)=$ $0.2]$. This means that specially prepared multilayer dielectric mirrors may be very effective for EUV at grazing angles.

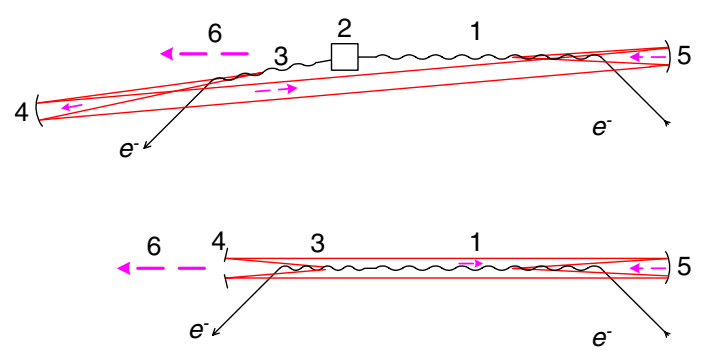

FIG. 6. Two schemes of the regenerative-amplifier FEL, exploiting electron outcoupling technique. Top: with $e$-beam bending. Bottom: with tapering. 1-main undulator, 2-achromatic bend, 3-outcoupling undulator section: bended (top) or tapered (bottom), 4 and 5-mirrors, 6-radiation from the main undulator. Radiation used for feedback is shown by red lines and small violet arrows.

In that context positive experience with multilayer focusing mirrors in the soft $\mathrm{x}$-ray region [23] is very encouraging.

Another way to eliminate the problem is to use the socalled electron outcoupling technique [24]. In this case the coherent radiation from the last section of the long undulator is used for feedback. This can be achieved in two ways [25]. Both schemes are shown in Fig. 6.

The first method uses an achromatic bend before the last undulator section. In this case, the radiation may be deflected from the main undulator axis, as shown in Fig. 6, top.

The second method (tapering) uses the last undulator section with a shorter period or a lower field amplitude. Consequently, the wavelength of radiation in the forward direction is shorter, according to Eq. (1). Therefore, the coherent radiation of the microbunched beam is synchronized with the undulator field only at some off-axis angle. The coherent radiation of the tapered section follows therefore a hollow angular distribution and can be recirculated using a hollow mirror (Fig. 6, bottom). The optimal period of the initial part of the main undulator may also be shorter than the regular value. Therefore, we plan to perform detailed calculations for this option.

\section{B. Consideration of an incoherent storage-ring-based source for EUV lithography}

Since EUV lithography does not demand particular coherence, one may be interested in the feasibility of a high-power storage ring EUV source. Such a source would have clear advantages of relative simplicity and technological maturity.

The total incoherent undulator radiation power may be quite high. According to Wiedemann [26],

$$
P(W)=6.336 E^{2}(\mathrm{GeV}) B_{0}{ }^{2}(\mathrm{kGs}) I(A) L(\mathrm{~m}),
$$

where $L$ is the undulator length and $I$ is the circulating current. Taking $L=30 \mathrm{~m}$ and $I=1 \mathrm{~A}$, for $E=$ $0.5-1.1 \mathrm{GeV}, B_{0}=5-9$ kGs (Figs. 1 and 2), we get 


$$
P(\text { total })=1.2-18 \mathrm{~kW} .
$$

This radiation has, however, a very broadband spectrum and its total power cannot be exploited. In particular, already at $d=2.7 \mathrm{~cm}(K=2)$ the intensity of the 9th (!) harmonic is nearly as strong as that of the fundamental. Since the spectral bandwidth for the EUV lithography applications is about 1\% [2], the above kilowatts are actually reduced to tens of Watts. This estimation is confirmed by the following calculation.

The average number of undulator radiation photons $n_{\mathrm{ph}}$ (per electron) at basic harmonic is given by the formula [26]

$$
n_{\mathrm{ph}}=\pi \alpha N(\Delta \omega / \omega) K^{2} /\left(1+K^{2} / 2\right) f^{2},
$$

where $\alpha$ is the fine structure constant $\alpha \sim 1 / 137, N$ is the number of undulator periods, $\Delta \omega / \omega$ is the bandwidth, and $f$ is the usual Bessel-function factor defined above in (5).

Multiplying this value by the electron flux $I / e$ and by the photon energy $E_{\mathrm{ph}}$ we finally get for the EUV power

$$
P=\pi \alpha N(\Delta \omega / \omega) f^{2} I \times\left(E_{\mathrm{ph}} / e\right) \times K^{2} /\left(1+K^{2} / 2\right) .
$$

As already mentioned above [2], for EUV lithography applications $\Delta \omega / \omega \sim 1 \%$. We have to illuminate some finite area and there are no requirements for the space coherence of this radiation. Therefore, we can use all photons from the spectrum, integrated over all angles within the bandwidth $\Delta \omega / \omega$. The entire angular span is essentially very narrow due to the high electron energies
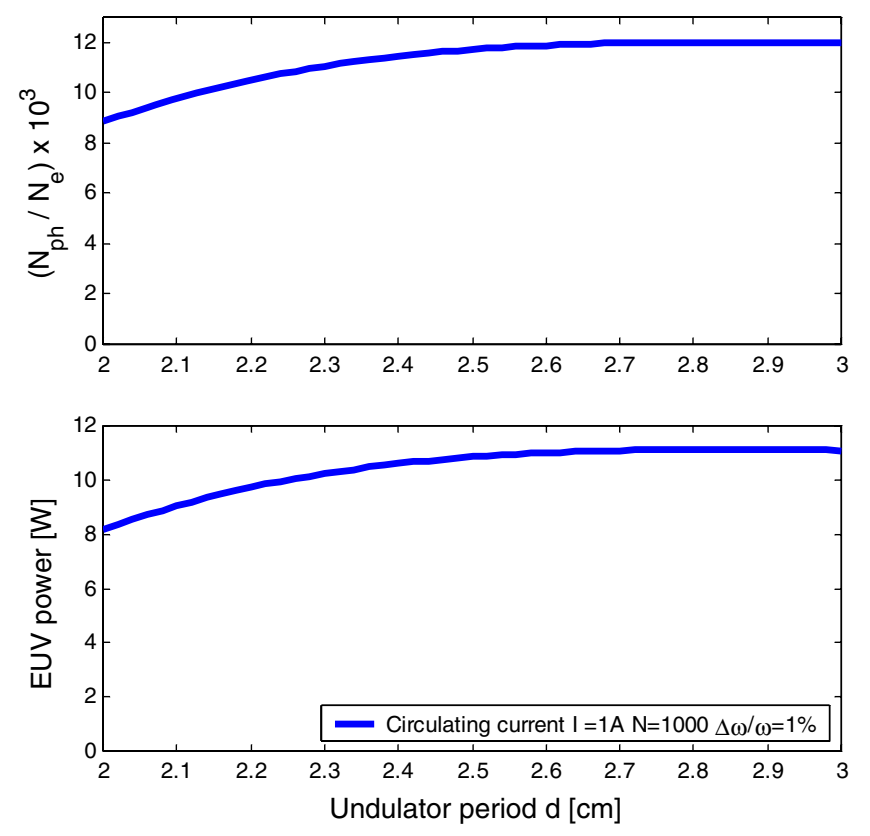

FIG. 7. EUV power of an incoherent storage-ring-based EUV source. Circulating current is $1 \mathrm{~A}$, number of periods $N=10^{3}$, and typical for EUV lithography bandwidth of $1 \%$. The output EUV power of Watt scale is of no interest in the context of highvolume manufacturing. involved: for $\gamma=1000-2000$ the opening angle of the radiation is of mrad scale even for the wavelength doubling, so even relatively long undulators do not obstruct the radiation. Consequently, the $1 / N$ factor (the spectrum width after the pinhole) does not matter, and the EUV power increases linearly with the undulator length, as stated in formula (8). Since $E_{\mathrm{ph}} / e$ is just photon energy in $\mathrm{eV}$ and $13.5 \mathrm{~nm}$ correspond to $92 \mathrm{eV}$, it is immediately seen that even for high circulating current of $1 \mathrm{~A}$ and long undulator $N=1000$, the output EUV power is of the order of Watts. The results of the calculation are shown in Fig. 7. We are therefore urged to conclude that incoherent storagering-based EUV radiation sources are of no interest for high-volume manufacturing.

\section{CONCLUSIONS}

We have shown that the use of a multiturn energyrecovery linac allows building a rather compact $40 \times 20 \mathrm{~m}^{2}$ EUV FEL for industrial lithography. The fact that a relatively low peak current value of $150 \mathrm{~A}$ is required reduces the coherent synchrotron radiation and other sources of the beam quality degradation. A relatively low average current of $10 \mathrm{~mA}$ and the advanced ERL magnetic system prevent instabilities. The use of the regenerative-amplifier FEL scheme may further improve the radiation parameters.

\section{ACKNOWLEDGMENTS}

We would like to thank all the colleagues who took part in the stimulating discussions during the FEL2010 conference at Malmo, Sweden. We would especially like to thank Dr. D. Douglas (JLab), Dr. E. Gluskin (ANL/XFEL), Dr. V. Litvinenko (BNL), Dr. B. Militsyn (ASTeC), Dr. D. Ratner (SLAC), Dr. G. Stupakov (SLAC), and Dr. A. Zholents (ANL).

[1] G. E. Moore, Electron Devices Meeting, 1975 International (1975), p. 11.

[2] B. Q. Wua and A. Kumar, J. Vac. Sci. Technol. 25, 1743 (2007); D. McGrath and M. LaPedus, EE Times 2011, http://www.eetimes.com/electronics-news/4213996/ Analysis-Litho-world-needs-a-shrink-

[3] C. Pagani, E. L. Saldin, E. A. Schneidmiller, and M. V. Yurkov, Nucl. Instrum. Methods Phys. Res., Sect. A 475, 391 (2001).

[4] N.A. Vinokurov et al., in Proceedings of the First International Particle Accelerator Conference, Kyoto, Japan (2010), p. 2427, http://accelconf.web.cern.ch/ AccelConf/IPAC10/papers/weoara03.pdf.

[5] D. Douglas, ICFA Beam Dynamics Newsletter 26, 40 (2001), http://icfa-usa.jlab.org/archive/newsletter/icfa_bd_ nl_26.pdf. 
[6] N. A. Vinokurov, ICFA Panel on Advanced and Novel Accelerators Newsletter (2009), http://www.nuclear.jp/ $\sim$ icfa/NewsLetter-Dec-09.pdf.

[7] J. Goldstein, D. Nguyen, and R. Sheffield, Nucl. Instrum. Methods Phys. Res., Sect. A 393, 137 (1997).

[8] S. V. Benson et al., in Proceedings of the First International Particle Accelerator Conference, Kyoto, Japan (Ref. [4]), p. 2302, http://accelconf.web.cern.ch/ AccelConf/IPAC10/papers/tupe074.pdf.

[9] P. Elleaume, J. Chavanne, and B. Faatz, Nucl. Instrum. Methods Phys. Res., Sect. A 455, 503 (2000).

[10] N. A. Vinokurov, Ph.D. thesis, Budker Institute Nuclear Physics, Novosibirsk, 1986.

[11] N. A. Vinokurov, O. A. Shevchenko, and V. G. Tcheskidov, in Proceedings of FEL2010, Malmo, Sweden (2010), THOC3, http://srv-fel-0.maxlab.lu.se/TOC/ THOC3.PDF.

[12] N.A. Vinokurov et al., in Proceedings of the 31st International Free Electron Laser Conference (FEL 09), Liverpool, UK (STFC Daresbury Laboratory, Warrington, 2009), p. 447.

[13] Z. Huang and K. J. Kim, Phys. Rev. ST Accel. Beams 10, 034801 (2007).

[14] D. Ratner et al., in Proceedings of the 31st International Free Electron Laser Conference (FEL 09), Liverpool, UK (Ref. [12]), p. 221.
[15] P. Emma (LCLS Commissioning Team), in Proceedings of the 23rd Particle Accelerator Conference, Vancouver, Canada, 2009 (IEEE, Piscataway, NJ, 2009), p. 3115.

[16] R. Bonifacio, C. Pellegrini, and L. Narducci, Opt. Commun. 50, 373 (1984).

[17] E. Gluskin et al., Nucl. Instrum. Methods Phys. Res., Sect. A 429, 358 (1999).

[18] M. Xie, Nucl. Instrum. Methods Phys. Res., Sect. A 445, 59 (2000).

[19] W. A. Barletta et al., Nucl. Instrum. Methods Phys. Res., Sect. A 618, 69 (2010).

[20] CVI Melles Griot (Albuquerque NM), http://www .cvimellesgriot.com/Products/Tunable-Laser-Line-Mirrors .aspx.

[21] D. H. Dowell et al., IEEE J. Quantum Electron. 27, 2613 (1991).

[22] L.D. Landau and E. M. Lifshitz, Electrodynamics of Continuous Media (Butterworth-Heinemann, London, 1984), 2nd ed., Sec. 86.

[23] A. Y. Lopatin et al., Tech. Phys. 55, 1018 (2010).

[24] N. G. Gavrilov et al., Nucl. Instrum. Methods Phys. Res., Sect. A 304, 63 (1991).

[25] G. N. Kulipanov et al., Nucl. Instrum. Methods Phys. Res., Sect. A 375, 576 (1996).

[26] H. Wiedemann, Particle Accelerator Physics II (SpringerVerlag, Berlin, 1999), Chap. 11. 